\title{
Assessment of Formalin Teratogenicity on Embryos of Pregnant
} Albino Rats

\author{
Original \\ Fardous A. Al- Kalaa ${ }^{1}$, Magdy M. H. El Bordiny ${ }^{2}$, Melad N. kelada ${ }^{1}$, \\ Article \\ Aliaa M. T. A. El-Alkamy ${ }^{l}$ \\ ${ }^{1}$ Human Anatomy and Embryology Department, ${ }^{2}$ clinical pathology Department, Faculty of \\ Medicine, Alexandria University
}

\begin{abstract}
Background: Formaldehyde (FA) is an economically important chemical that is commonly used in the production of industrial, laboratory, household, and cosmetic products. FA is a potential carcinogen and mutagen.

Exposure to formaldehyde is common especially among medical and paramedical personnel as it is widely used disinfectant. The exposure becomes maximal and semi- obligatory among workers and personnel of anatomy departments in the different faculties of medicine in a lot of countries and Egypt among; it is the main substance used for cadavers and human parts preservations.

Workers and staff of anatomy departments among those with the highest possible formalin exposure with the risk to experience part or most of its possible serious hazardous health effects. This attracts our attention and necessitates a careful study for the possible effects of formalin on the embryos of exposed mothers.

Objectives: This study was designed to evaluate the teratogenicity of formaldehyde in experimental animals exposed to FA. Methods: The study included fifty adult female albino Mice, that were divided into five groups ; (Ia): Negative control group received distilled water, $(\mathrm{Ib})$ : Positive control: received oral colchicines $2 \mathrm{mg} / \mathrm{kg}$ /day on gestational days 6-13, Group II, III and IV: received formaldehyde orally in gestational days 6-13 with concentrations 7,14 and $21 \mathrm{mg} / \mathrm{kg} / \mathrm{day}$ respectively. Pregnant females were sacrificed on $18^{\text {th }}$ gestational day. The uterine horns were examined for resorption sites, alive or dead fetuses. Extracted fetuses were examined for external developmental abnormalities and stained for skeletal malformations.

Results: Decrease in the body weight of the newborns, Increase frequency of abortion and Fetuses mortality and there were some anomalies among the newborn mice which included umbilical hernia, subcutaneous bleeding, ear deformity, delayed ossification of skull bones and Caudal vertebrae were unossified or showed very small ossification centre.

Conclusion: The pervious findings suggest that formaldehyde is a teratogenic substance.
\end{abstract}

Received: 24 July 2019, Accepted: 01 September 2019

Key Words: Formaldehyde; genotoxic; pregnancy; skeletal defects; teratogenic.

Corresponding Author: Melad Naim Bushra Kelada, MD, Anatomy and Embryology Department, Faculty of Medicine, Alexandria University, Egypt, Tel.: +20 1226636044, E-mail: meladkelada@ymail.com

ISSN: 1110-0559, Vol. 43, No. 1

\section{INTRODUCTION}

Formaldehyde (FA) is a gaseous substance found in many outdoors and indoors sources. Outdoors, major sources of FA include factories especially power factories, and emissions form vehicles exhaust. Indoors include release from different building materials and tobacco smoke. It is also present in many food stuffs, either naturally or as a result of contamination ${ }^{[1]}$.

It has so many medical uses as treatment of hemorrhagic cystitis, hydatid cyst surgery and very commonly as a potent disinfectant.

FA is highly toxic regardless of method of exposure. It is also a sever irritant to the skin, eyes, mouth, nose and upper respiratory $\operatorname{tract}^{[2]}$.

It affects CNS and its toxic effects include excessive marked fatigue, headache, memory difficulties, irritability and unstable mood. Convulsions can occur and sometimes accompanied by an olfactory $\operatorname{aura}^{[3]}$.

FA reported to be cardio toxic. It can produce bradycardia, decreased cardiac output, coma and death may occur. Some cases of formalin induced A - V arrhythmia has been reported. Formalin also has a direct effect on vascular smooth muscles so it induces arterial hypotension ${ }^{[4]}$.

FA increases the incidence of embryo mortality; it is also a teratogenic agent where it increases fetal anomalies as cryptochordism and aberrant ossification centers. And at the molecular level it decrease concentrations of ascorbic acid; and cause abnormalities in enzymes of mitochondria, lysosomes, and endoplasmic reticulum ${ }^{[5]}$.

FA also proved to be genotoxic where it is found to increases DNA-protein cross-links and can causes 
significant increases in chromosomal aberrations and chromosomal breakage ${ }^{[6,7]}$.

FA was classified as a Class I carcinogen as it is strongly related to nasopharyngeal cancer in humans ${ }^{[8]}$.

FA was reclassified as a factor that causes leukemia because of the increasing evidence that it can induces genetic changes in blood cells. As it has been found to cause aneuploidy of chromosomes 7 and 8 in hematopoietic progenitor cells of exposed workers that are characteristic of myeloid leukemia and myelodysplastic syndromes ${ }^{[9,10]}$.

FA causes genetic damage directly in the nasal sinus, via formation of DNA-protein cross-links, but less is known about how it induces chromosomal abnormalities and other genetic changes characteristic of leukemia within the hematopoietic system ${ }^{[11]}$.

\section{OBJECTIVES}

The objective of the present study was to assess the developmental toxicity and possible congenital malformations induced by Formaldehyde, in CD-1 mice, thereby avoiding the teratogenic potential of FA during pregnancy.

\section{METHODS}

The study included fifty adult female albino Mice, that were divided into five groups 10 each; (Ia): Negative control group received distilled water, (Ib): Positive control: received oral colchicines $2 \mathrm{mg} / \mathrm{kg} /$ day on gestational days 6-13, Group II, III and IV: received formaldehyde orally in gestational days 6-13 with concentrations 7, 14 and $21 \mathrm{mg} /$ $\mathrm{kg} /$ day respectively ${ }^{[12]}$. Pregnant females were sacrificed on $18^{\text {th }}$ gestational day. The uterine horns were examined for resorption sites, alive or dead fetuses. Extracted fetuses were examined for external developmental abnormalities and stained for skeletal malformations.

For skeletal examination $50 \%$ of the fetuses undergo clearing the soft tissues by $1 \%$ potassium hydroxide $(\mathrm{KOH})$ solution was for 5-7 days, the fetuses remained in the $\mathrm{KOH}$ solution until they were distinctly transparent and the lightcolored skeletal system was well visible. The skeleton was stained in a dilute alkaline solution of Alizarin Red S. ${ }^{[13,14]}$.

Colchicine is used as a positive control to assure the performance of in vivo micronucleus test. It produces detectable increase in micronucleus frequency over the spontaneous level ${ }^{[12]}$.

\section{Statistical Analysis of the Data}

Data were fed to the computer and analyzed using IBM SPSS software package version 20.0. Both statistical analysis and tabulation were done according to Altman. Differences between 2 continuous variables were compared with student " $t$ " test. Differences between 3 or more continuous variables were compared with one way analysis of variance (ANOVA), using Scheffe's method for multiple comparisons. Differences between proportions were compared with the Chi- squared and Fisher's exact test. The level of significance was set at $p \leq 0.05^{[15,16]}$.

\section{RESULTS}

There was a statistically significant reduction in the amount of food consumption in positive control group (group Ib) and in the three FA groups (II, III and IV) compared to their corresponding negative control groups. This reduction appeared on day 9 of gestation and progressed through the gestational period. On gestational day 18 the food consumption reduction in group IV was $42 \%$ compared to their corresponding negative control; (Table 1).

Regarding the weight gain, there was a significant decrease in dams' weight gain between day 6 and day 18 in groups Ib, III and IV, while the decrease in group II was insignificant compared to negative control (group Ia); (Table 2).

Signs of toxicity including lethargy, decrease in motor activity, and loss of appetite were noted in dams of treated with FA compared to both colchicine and negative control groups. These effects appeared on day 10 of gestation (day5 of treatment) and progressed throughout the treatment period. These signs were more obvious in group IV (FA $21 \mathrm{mg} / \mathrm{kg} / \mathrm{d}$ ).

There was significant increase in abortion rate in positive control group (group IB) by $60 \%$. The abortion rate increased insignificantly in groups II, III and IV (Table 3).

There was insignificant increase in the number of early resorptions in groups II, III and IV in comparison to negative control group (Table 4).

On the other hand, administration of FA at a dose of 21 $\mathrm{mg} / \mathrm{kg} / \mathrm{d}$ on gestational days 6-13 in group IV resulted in significant increase in late resorption sites compared to the negative control, while the increase group II and III was insignificant (Table 4, Figure 1).

The rate of live fetuses was significantly decreased in groups III and IV compared to the negative control. Also, the rate of dead fetuses was significantly increased in the same groups. However, administration of FA $7 \mathrm{mg} / \mathrm{kg} / \mathrm{d}$ at group II did not cause any significant change in the rate of live and dead fetuses (Table 4).

It seems to be the same trend towards increased resorptions and deceased live fetuses with increasing the FA dose (Figures 7-10).

The average fetal weight was significantly decreased in a dose-dependent manner in groups III and IV (FA 14 and $21 \mathrm{mg} / \mathrm{kg} / \mathrm{d}$ respectively); however no significant change was noticed in group II (FA $7 \mathrm{mg} / \mathrm{kg} / \mathrm{d}$ ) (Table 5).

Also, the placental weight decreased in a similar pattern; it was significantly decreased in groups III and IV, but group II showed no significant change compared to negative control (Table 3).

Fetuses with macroscopic anomalies such as Meningeocoele were seen in $5.26 \%$ of group IV only, 
which was insignificant. Open eye lids were observed in $9.23 \%$ and $15.78 \%$ in groups III and IV respectively which was significant (Figure 2, Table 4).

Ophthalmic anomalies, namely, an-ophthalmia $(1.31 \%$, $3.07 \%$, and $13.15 \%$ ) in groups II,III and IV respectively and micro-ophthalmia $(2.28 \%, 1.95 \%$ and $15.78 \%)$ in the three treatment groups respectively were observed (Figures 4,5, Table 6).

Ear abnormalities were observed such as absent ear (7.89\% in group IV), displaced ear $(3.94 \%, 3.07 \%$ and $18.42 \%$ in groups II, III and IV respectively) and deformed ear (1.53\% and $10.53 \%$ in groups III and IV) (Table 6).

Abdominal wall defects such as omphalocele (3.07 and $10.52 \%$ in group III and IV) and umbilical hernia (3.95\%, $10.77 \%$ and $18.42 \%$ in groups II, III and IV) were evident. (Figures 3,4, Table 6).
Also subcutaneous hemorrhage in the cranial, abdominal, thoracic regions and tail were observed in the fetuses of females treated with FA compared to their corresponding negative control group (Figures 3,4,5, Table 6).

\section{1- Skeletal Abnormalities}

Alizarin red S stained skeletons of fetuses revealed many anomalies such as reduced ossification of frontal, parietal, interparietal, and suboccipital bones of skull, reduced, absent, displaced vertebral centra in thoracic and lumbar regions, reduction ,absence of caudal vertebrae, reduced pelvic elements, and absence of carpals, metacarpals, tarsals, metatarsals, and phalanges. (Figures 6-12, Table 7).

The occurrence of skeletal anomalies was proportionate to the dose (Table 7, Figures 6-12).

Table 1: Comparison between the four studied groups as regards food consumption of dams throughout the treatment period

\begin{tabular}{|c|c|c|c|c|c|}
\hline \multirow{2}{*}{$\begin{array}{l}\text { Mean food } \\
\text { consumption } \\
\text { (mg/day) }\end{array}$} & \multicolumn{2}{|c|}{ Control (Group I) } & \multirow{2}{*}{$\begin{array}{c}\text { Group II } \\
\text { FA }(7 \mathrm{mg} / \mathrm{kg} / \mathrm{d}) \\
(\mathrm{n}=10)\end{array}$} & \multirow{2}{*}{$\begin{array}{c}\text { Group III } \\
\text { FA }(14 \mathrm{mg} / \mathrm{kg} / \mathrm{d}) \\
(\mathrm{n}=10)\end{array}$} & \multirow{2}{*}{$\begin{array}{l}\text { Group IV FA } \\
(21 \mathrm{mg} / \mathrm{kg} / \mathrm{d}) \\
(\mathrm{n}=10)\end{array}$} \\
\hline & $\begin{array}{c}\text { Negative (IA) } \\
(\mathrm{n}=10)\end{array}$ & $\begin{array}{c}\text { Positive } \\
\text { (IB) }(\mathrm{n}=10)\end{array}$ & & & \\
\hline GD 1 & $356.6 \pm 4.79$ & $362.0 \pm 15.13$ & $348.2 \pm 1.81$ & $356.20 \pm 3.26$ & $348.30 \pm 4.11$ \\
\hline$P$ & & 0.501 & 0.109 & 1.000 & 0.116 \\
\hline GD6 & $368.2 \pm 2.7$ & $367.4 \pm 13.7$ & $360.4 \pm 1.84$ & $374.5 \pm 4.17$ & $374.5 \pm 4.06$ \\
\hline$P$ & & 0.999 & 0.096 & 0.252 & 0.252 \\
\hline GD9 & $396.0 \pm 5.54$ & $251.4 \pm 9.44$ & $377.6 \pm 1.96$ & $233.6 \pm 4.48$ & $244.4 \pm 7.38$ \\
\hline$P$ & & $<0.001^{*}$ & $<0.001^{*}$ & $<0.001^{*}$ & $<0.001^{*}$ \\
\hline GD12 & $401.4 \pm 7.32$ & $201.6 \pm 5.6$ & $416.6 \pm 2.55$ & $261.6 \pm 5.1$ & $193.0 \pm 17.35$ \\
\hline$P$ & & $<0.001^{*}$ & $0.005^{*}$ & $<0.001^{*}$ & $<0.001^{*}$ \\
\hline GD15 & $429.4 \pm 3.03$ & $190.6 \pm 5.64$ & $447.4 \pm 5.72$ & $251.1 \pm 2.23$ & $176.3 \pm 27.55$ \\
\hline$P$ & & $<0.001^{*}$ & $0.026^{*}$ & $<0.001^{*}$ & $<0.001^{*}$ \\
\hline GD18 & $431.6 \pm 13.44$ & $170.6 \pm 4.6$ & $449.0 \pm 1.49$ & $234.9 \pm 3.35^{\#}$ & $164.78 \pm 26.2^{\#}$ \\
\hline$P$ & & $<0.001^{*}$ & $0.035^{*}$ & $<0.001^{*}$ & $<0.001^{*}$ \\
\hline
\end{tabular}

\#: $(\mathrm{n}=9)$ - $\mathrm{p}: p$ value for comparing between negative control and each other groups - *: Statistically significant at $p \leq 0.05$

Table 2: Comparison between the four studied groups as regards mean weight gain of dams throughout the treatment period

\begin{tabular}{|c|c|c|c|c|c|}
\hline \multirow{2}{*}{$\begin{array}{c}\text { Mean weight } \\
\text { gain }(\mathrm{g})\end{array}$} & \multicolumn{2}{|c|}{ Control (Group I) } & \multirow{2}{*}{$\begin{array}{c}\text { Group II } \\
\text { FA }(7 \mathrm{mg} / \mathrm{kg} / \mathrm{d}) \\
\quad(\mathrm{n}=10)\end{array}$} & \multirow{2}{*}{$\begin{array}{c}\text { Group III } \\
\text { FA }(14 \mathrm{mg} / \mathrm{kg} / \mathrm{d}) \\
(\mathrm{n}=10)\end{array}$} & \multirow{2}{*}{$\begin{array}{c}\text { Group IV FA } \\
(21 \mathrm{mg} / \mathrm{kg} / \mathrm{d}) \\
(\mathrm{n}=10)\end{array}$} \\
\hline & $\begin{array}{l}\text { Negative (IA) } \\
\quad(\mathrm{n}=10)\end{array}$ & $\begin{array}{c}\text { Positive } \\
\text { (IB) }(n=10)\end{array}$ & & & \\
\hline GD1 - GD6 & $2.70 \pm 1.16$ & $1.80 \pm 0.79$ & $2.30 \pm 1.06$ & $2.80 \pm 2.80$ & $2.30 \pm 0.82$ \\
\hline$P$ & & 0.071 & 0.410 & 0.563 & 0.362 \\
\hline GD6 - GD9 & $2.50 \pm 1.18$ & $-1.0 \pm 1.15$ & $2.10 \pm 0.99$ & $1.10 \pm 0.57$ & $0.60 \pm 0.52$ \\
\hline$P$ & & $<0.001^{*}$ & 0.406 & $0.008^{*}$ & $0.001^{*}$ \\
\hline GD9 - GD12 & $1.30 \pm 0.42$ & $-1.0 \pm 1.63$ & $2.0 \pm 0.67$ & $0.50 \pm 0.85$ & $0.50 \pm 0.97$ \\
\hline$P$ & & $0.013^{*}$ & $0.015^{*}$ & $0.018^{*}$ & $0.010^{*}$ \\
\hline GD12 - GD15 & $3.90 \pm 0.52$ & $-2.20 \pm 0.79$ & $2.90 \pm 6.70$ & $2.70 \pm 0.95$ & $1.10 \pm 1.52$ \\
\hline$P$ & & $<0.001^{*}$ & 0.117 & $0.004^{*}$ & $<0.001^{*}$ \\
\hline GD15 - GD18 & $5.30 \pm 0.92$ & $-3.20 \pm 1.23$ & $4.50 \pm 2.00$ & $2.50 \pm 1.08 \#$ & $0.89 \pm 2.85$ \\
\hline$P$ & & $<0.001^{*}$ & 0.536 & $<0.001^{*}$ & $<0.001^{*}$ \\
\hline Overall (6-18) & $13.0 \pm 1.55$ & $-7.40 \pm 3.57$ & $11.50 \pm 7.24$ & $6.80 \pm 1.40^{\#}$ & $3.78 \pm 2.99$ \\
\hline$P$ & & $<0.001^{*}$ & 0.790 & $<0.001^{*}$ & $<0.001^{*}$ \\
\hline
\end{tabular}

$\#:(\mathrm{n}=9)-\mathrm{p}: p$ value for comparing between negative control and each other groups - *: Statistically significant at $p \leq 0.05$ 
Table 3: Comparison between the four studied groups according to mortality and abortion rate

\begin{tabular}{|c|c|c|c|c|c|}
\hline & \multicolumn{2}{|c|}{ Control (Group I) } & \multirow{2}{*}{$\begin{array}{c}\text { Group II } \\
\text { FA }(7 \mathrm{mg} / \mathrm{kg} / \mathrm{d}) \\
(\mathrm{n}=10)\end{array}$} & \multirow{2}{*}{$\begin{array}{c}\text { Group III } \\
\text { FA }(14 \mathrm{mg} / \mathrm{kg} / \mathrm{d}) \\
(\mathrm{n}=10)\end{array}$} & \multirow{2}{*}{$\begin{array}{c}\text { Group IV } \\
\text { FA }(21 \mathrm{mg} / \mathrm{kg} / \mathrm{d}) \\
(\mathrm{n}=10)\end{array}$} \\
\hline & $\begin{array}{l}\text { Negative (IA) } \\
(\mathrm{n}=10)\end{array}$ & $\begin{array}{c}\text { Positive (IB) } \\
(\mathrm{n}=10)\end{array}$ & & & \\
\hline abortion & $0(0.0)$ & $6(60.0 \%)$ & $2(20.0 \%)$ & $2(20.0 \%)$ & $2(20.0 \%)$ \\
\hline mortality & $0(0.0)$ & $4(40.0 \%)$ & $0(0.0)$ & $0(0.0)$ & $1(10.0 \%)$ \\
\hline$p$ & & $p<0.001^{*}$ & $p=0.474$ & $p=0.474$ & $p=0.201$ \\
\hline
\end{tabular}

*: Statistically significant at $p \leq 0.05$ - $\mathrm{p}: p$ value for comparing between negative control and each other groups - AB: Abortion.

Table 4: Comparison between the four studied groups according to developmental toxicity on gestational day 18

\begin{tabular}{|c|c|c|c|c|}
\hline & $\begin{array}{c}\text { Control Group I } \\
\text { Negative (IA) } \\
(\mathrm{n}=10)\end{array}$ & $\begin{array}{c}\text { Group II } \\
\text { FA }(7 \mathrm{mg} / \mathrm{kg} / \mathrm{d}) \\
(\mathrm{n}=10)\end{array}$ & $\begin{array}{c}\text { Group III } \\
\text { FA }(14 \mathrm{mg} / \mathrm{kg} / \mathrm{d}) \\
(\mathrm{n}=10)\end{array}$ & $\begin{array}{c}\text { Group IV } \\
\text { FA }(21 \mathrm{mg} / \mathrm{kg} / \mathrm{d}) \\
(\mathrm{n}=10)\end{array}$ \\
\hline No. of implants & $9.70 \pm 0.95$ & $9.50 \pm 1.20$ & $8.13 \pm 3.09$ & $5.43 \pm 1.27$ \\
\hline$p$ & & 0.712 & 0.169 & $0.001^{*}$ \\
\hline Early resorptions & $0.20 \pm 0.42$ & $0.20 \pm 0.42$ & $0.50 \pm 0.53$ & $0.67 \pm 0.71$ \\
\hline$p$ & & 1.000 & 0.170 & 0.102 \\
\hline Late resorptions & $0.0 \pm 0.0$ & $0.20 \pm 0.42$ & $0.10 \pm 0.32$ & $1.11 \pm 0.78$ \\
\hline$p$ & & 0.146 & 0.317 & $0.001^{*}$ \\
\hline Live fetuses & $10.20 \pm 0.79$ & $9.50 \pm 1.20$ & $6.80 \pm 3.71$ & $3.11 \pm 1.90$ \\
\hline$p$ & & 0.194 & $0.001^{*}$ & $<0.001^{*}$ \\
\hline Dead fetuses & $0.0 \pm 0.0$ & $0.0 \pm 0.0$ & $0.70 \pm 0.82$ & $1.11 \pm 0.78$ \\
\hline$p$ & & 1.000 & $0.013^{*}$ & $0.001^{*}$ \\
\hline Sex ratio $(\mathrm{M}: \mathrm{F})$ & $1.03 \pm 0.34$ & $1.10 \pm 0.41$ & $1.24 \pm 0.23$ & $1.0 \pm 0.27$ \\
\hline$p$ & & 0.639 & 0.191 & 0.848 \\
\hline
\end{tabular}

p: $p$ value for comparing between negative control and each other groups - *: Statistically significant at $p \leq 0.05$

Table 5: Comparison between the four studied groups according to fetal and placental weights

\begin{tabular}{|c|c|c|c|c|}
\hline & $\begin{array}{l}\text { Negative Control Group (IA) } \\
\qquad(\mathrm{n}=97)\end{array}$ & $\begin{array}{l}\text { Group II FA }(7 \mathrm{mg} / \mathrm{kg} / \mathrm{d}) \\
\qquad(\mathrm{n}=76)\end{array}$ & $\begin{array}{l}\text { Group III FA }(14 \mathrm{mg} / \mathrm{kg} / \mathrm{d}) \\
(\mathrm{n}=65)\end{array}$ & $\begin{array}{c}\text { Group IV FA }(21 \mathrm{mg} / \mathrm{kg} / \mathrm{d}) \\
(\mathrm{n}=38)\end{array}$ \\
\hline $\begin{array}{l}\text { Fetal body weight }(\mathrm{g}) \\
\quad(\text { Mean } \pm \text { SD) }\end{array}$ & $0.95 \pm 0.16$ & $1.0 \pm 0.13$ & $0.80 \pm 0.04$ & $0.48 \pm 0.03$ \\
\hline$P$ & & 0.832 & $0.039^{*}$ & $<0.001^{*}$ \\
\hline $\begin{array}{l}\text { Placental weight (g) } \\
\quad(\text { Mean } \pm \text { SD) }\end{array}$ & $0.20 \pm 0.0$ & $0.20 \pm 0.01$ & $0.17 \pm 0.01$ & $0.14 \pm 0.02$ \\
\hline$P$ & & 0.586 & $<0.001^{*}$ & $<0.001^{*}$ \\
\hline
\end{tabular}

p: $p$ value for comparing between negative control and each other groups - *: Statistically significant at $p \leq 0.05$ 
Table 6: The incidence of external abnormalities in mouse fetuses after maternal exposure to FA

\begin{tabular}{|c|c|c|c|c|}
\hline $\begin{array}{l}\text { Number of fetuses } \\
\text { affected (percentage) }\end{array}$ & $\begin{array}{l}\text { Negative Control } \\
\text { Group IA }(\mathrm{n}=97)\end{array}$ & $\begin{array}{c}\text { Group II (7mgFA } / \mathrm{kg} / \mathrm{d}) \\
(\mathrm{n}=76)\end{array}$ & $\begin{array}{l}\text { Group III }(14 \mathrm{mgFA} / \mathrm{kg} / \mathrm{d}) \\
\qquad(\mathrm{n}=65)\end{array}$ & $\begin{array}{c}\text { Group IV (21mgFA/kg /d) } \\
(\mathrm{n}=38)\end{array}$ \\
\hline Meningeocoele & $0(0)$ & $0(0)$ & $0(0)$ & $2(5.26)$ \\
\hline$P$ & & - & - & 0.078 \\
\hline Open eye lid & $0(0)$ & $2(2.63)$ & $6(9.23)$ & $6(15.78)$ \\
\hline$P$ & & 0.192 & $0.004^{*}$ & $<0.001^{*}$ \\
\hline Anophthalmia & $0(0)$ & $1(1.31)$ & $2(3.07)$ & $5(13.15)$ \\
\hline$P$ & & 0.439 & 0.159 & $0.001^{*}$ \\
\hline Microphthalmia & $0(0)$ & $3(2.28)$ & $3(1.95)$ & $6(15.78)$ \\
\hline$P$ & & 0.083 & 0.063 & $<0.001^{*}$ \\
\hline Absent ear & $0(0)$ & $0(0)$ & $0(0)$ & $3(7.89)$ \\
\hline$P$ & & - & - & $0.021^{*}$ \\
\hline Displaced ear & $1(1.03)$ & $3(3.94)$ & $2(3.07)$ & $7(18.42)$ \\
\hline$P$ & & 0.321 & 0.565 & $0.001^{*}$ \\
\hline Deformed ear & $0(0)$ & $0(0)$ & $1(1.53)$ & $4(10.53)$ \\
\hline$P$ & & - & 0.401 & $0.006^{*}$ \\
\hline Omphalocele & $0(0)$ & $0(0)$ & $2(3.07)$ & $4(10.53)$ \\
\hline$P$ & & - & 0.159 & $0.006^{*}$ \\
\hline Umbilical hernia & $2(2.06)$ & $3(3.95)$ & $7(10.77)$ & $7(18.42)$ \\
\hline$P$ & & 0.655 & $0.031^{*}$ & $0.002^{*}$ \\
\hline \multicolumn{5}{|l|}{ Subcutaneus hemorrhage } \\
\hline At the Head and neck & $1(1.03)$ & $3(3.94)$ & $4(6.15)$ & $8(21.05)$ \\
\hline$P$ & & 0.321 & 0.158 & $<0.001^{*}$ \\
\hline At the Abdomen & $2(2.06)$ & $2(2.63)$ & $5(7.69)$ & $10(26.31)$ \\
\hline$P$ & & 1.000 & 0.118 & $<0.001^{*}$ \\
\hline At the Thorax & $1(1.03)$ & $1(1.31)$ & $0(0)$ & $4(10.52)$ \\
\hline$P$ & & 1.000 & - & $0.022^{*}$ \\
\hline At the Tail & $0(0)$ & $0(0)$ & $0(0)$ & $3(7.89)$ \\
\hline$P$ & & _- & - & 0.067 \\
\hline
\end{tabular}

$\mathrm{n}=$ number of fetuses in each group - $\mathrm{p}: p$ value for comparing between negative control and each other groups - *: Statistically significant at $p \leq 0.05$

Table 7: The incidence of skeletal abnormalities in mouse fetuses after maternal exposure to Formaldehyde (FA)

\begin{tabular}{|c|c|c|c|c|}
\hline $\begin{array}{c}\text { Number of fetuses affected } \\
\text { (percentage) }\end{array}$ & $\begin{array}{l}\text { Negative Control Group IA } \\
\qquad(\mathrm{n}=97)\end{array}$ & $\begin{array}{c}\text { Group II (7mgFA/kg /d) } \\
\qquad(\mathrm{n}=76)\end{array}$ & $\begin{array}{l}\text { Group III (14mgFA/kg /d }) \\
\qquad(\mathrm{n}=65)\end{array}$ & $\begin{array}{l}\text { Group IV (21 mg FA/kg /d) } \\
\qquad(\mathrm{n}=38)\end{array}$ \\
\hline $\begin{array}{l}\text { Total fetuses with different } \\
\text { skeletal anomalies }\end{array}$ & $0(0)$ & $17(22.37)$ & $23(35.38)$ & $19(50)$ \\
\hline$p$ & & $<0.001^{*}$ & $<0.001^{*}$ & $<0.001^{*}$ \\
\hline Skull anomalies & $0(0)$ & $13(17.11)$ & $16(24.61)$ & $15(39.47)$ \\
\hline$p$ & & $<0.001^{*}$ & $<0.001^{*}$ & $<0.001^{*}$ \\
\hline $\begin{array}{l}\text { Vertebral column } \\
\text { Anomalies }\end{array}$ & $0(0)$ & $0(0)$ & $3(4.61)$ & $4(10.53)$ \\
\hline$p$ & & - & 0.063 & $0.006^{*}$ \\
\hline $\begin{array}{l}\text { Caudal vertebrae } \\
\text { anomalies }\end{array}$ & $0(0)$ & $4(5.26)$ & $10(15.38)$ & $3(7.89)$ \\
\hline$p$ & & $0.036^{*}$ & $<0.001^{*}$ & $0.021^{*}$ \\
\hline \multirow[t]{2}{*}{$\begin{array}{l}\text { Carpals, metacarpals, tarsals, } \\
\text { metatarsals, and phalanges } \\
\text { anomalies }\end{array}$} & $0(0)$ & $14(18.42)$ & $17(26.15)$ & $18(47.37)$ \\
\hline & & $<0.001^{*}$ & $<0.001^{*}$ & $<0.001^{*}$ \\
\hline
\end{tabular}

p: $p$ value for comparing between negative control and each other groups - *: Statistically significant at $p \leq 0.05$ 


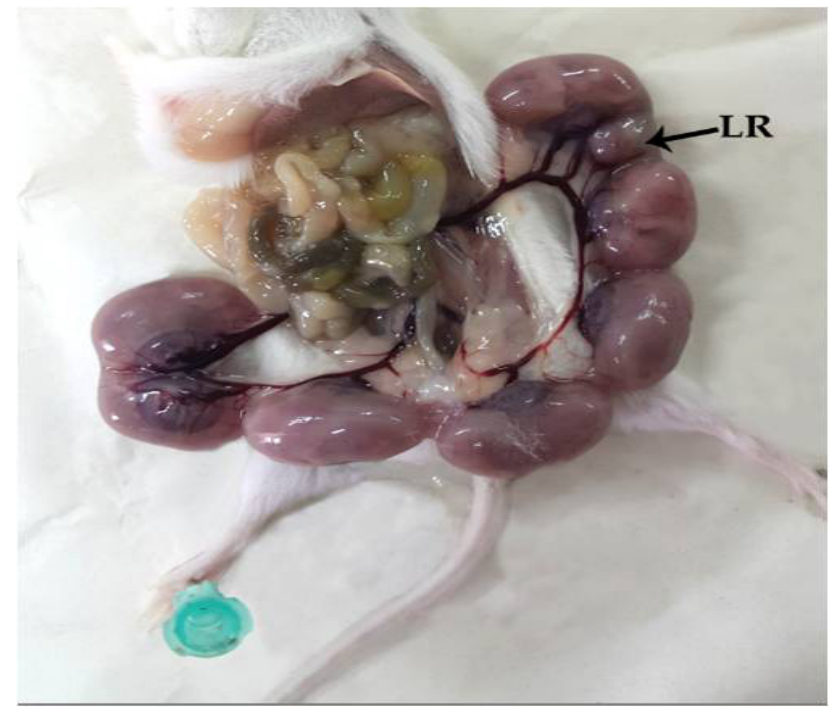

Fig. 1: Photograph of the uterus of a female mouse group IV on gestational day 18 showing seven fetuses and late resorption site (LR)inside the gestational sacs.

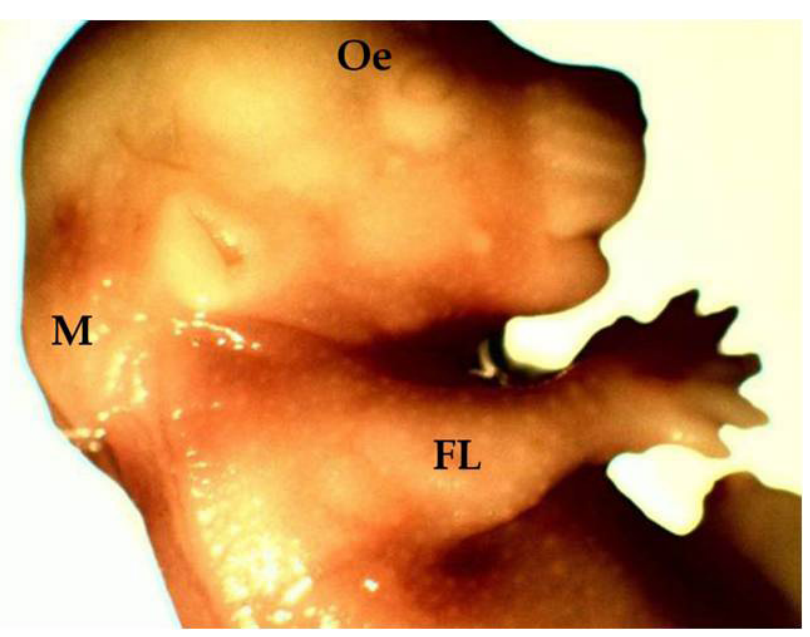

Fig. 2: Micrograph of mouse fetus of group IV taken by stereomicroscope (SZ 61) showing meningeocoele (M) and open eye lid (oe). FL: Fore limb. (X 10)

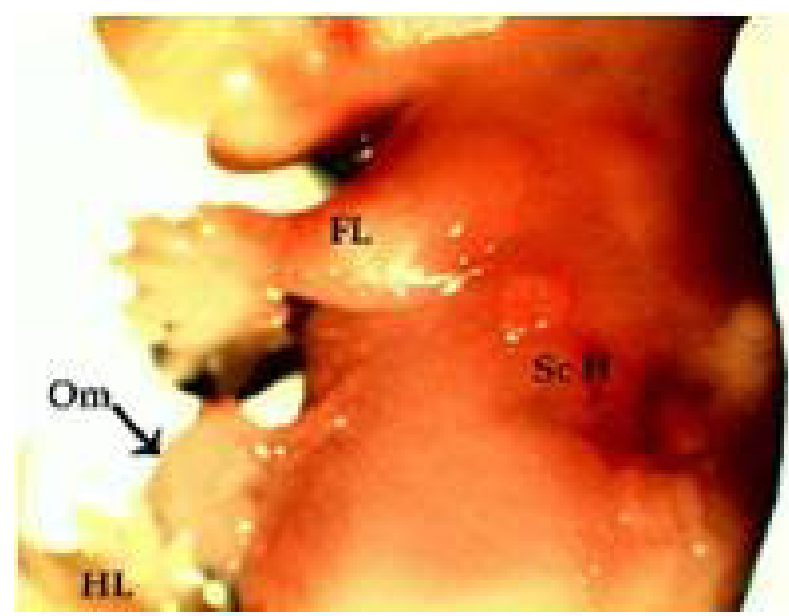

Fig. 3: Micrograph of GD 18 mouse fetus of group IV taken by stereomicroscope (SZ 61) showing omphalocele (Om) and subcutaneous hemorrhage in thoraco-abdominal region $(\mathrm{Sc} \mathrm{H})$. FL:Fore limb. HL: Hind limb. (X 10)

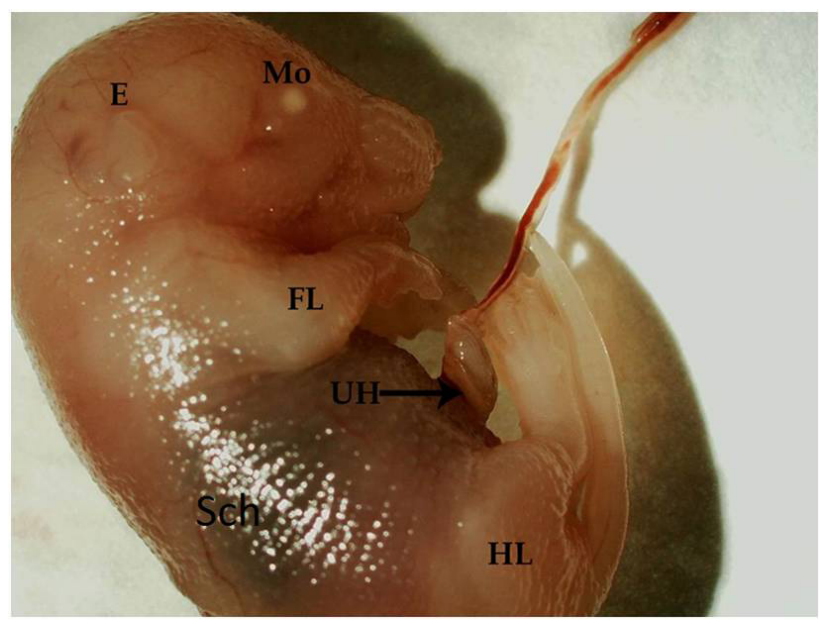

Fig. 4: Micrograph of gestational day 18 mouse fetus of group III taken by stereomicroscope (SZ 61) showing microphthalmia (Mo) and umbilical hernia (UH) and a large area of abdominal subcutaneous hemorrhage (Sch) . E: ear. FL:Fore limb. HL: Hind limb. (X 8)

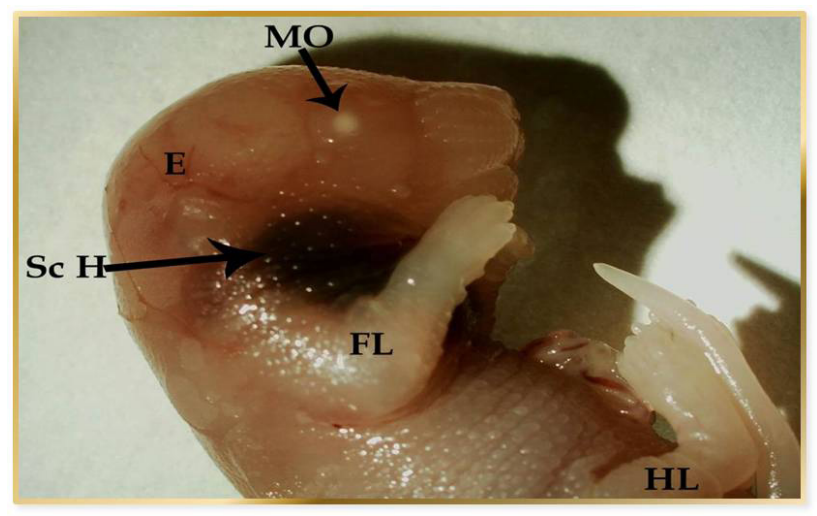

Fig. 5: Micrograph of GD 18 mouse fetus of group IV taken by stereomicroscope (SZ 61) showing microphthalmia (Mo) and subcutaneous hemorrhage in neck region $(\mathrm{Sc} \mathrm{H}) . \mathrm{E}$ : ear. FL Forelimb. HL: Hind limb. (X 8)

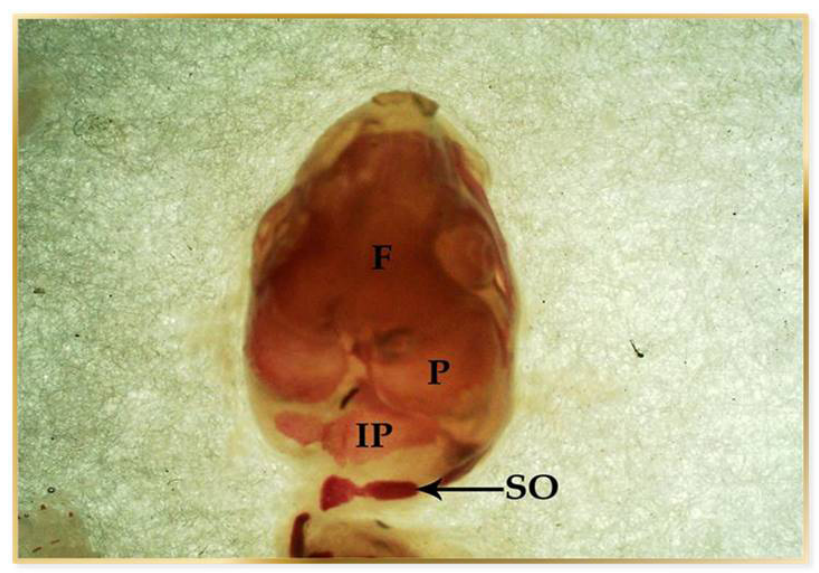

Fig. 6: Photomicrograph of fetal skull stained with alizarin red stain of negative control mouse fetus, dorsal view taken by stereomicroscope (SZ 61) showing: normal ossification of skull bones. F: frontal bone P: parietal bone IP: Interparietal bone. SO: suboccipital. (X 10) 


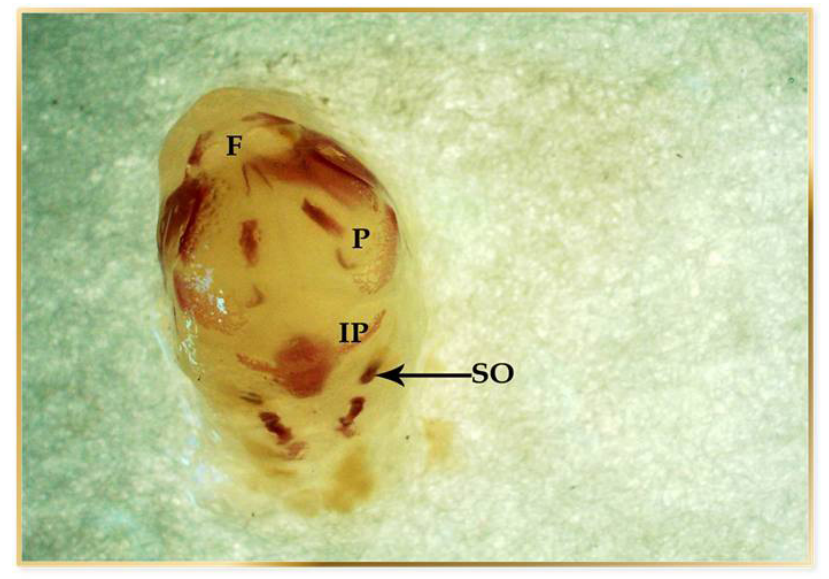

Fig. 7: Photomicrograph of fetal skull stained with alizarin red stain of group IV mouse fetus, dorsal view taken by stereomicroscope (SZ 61) showing: poor ossification of skul bones. F: frontal bone P: parietal bone. IP: Interparietal bone. SO: suboccipital.(X 10)

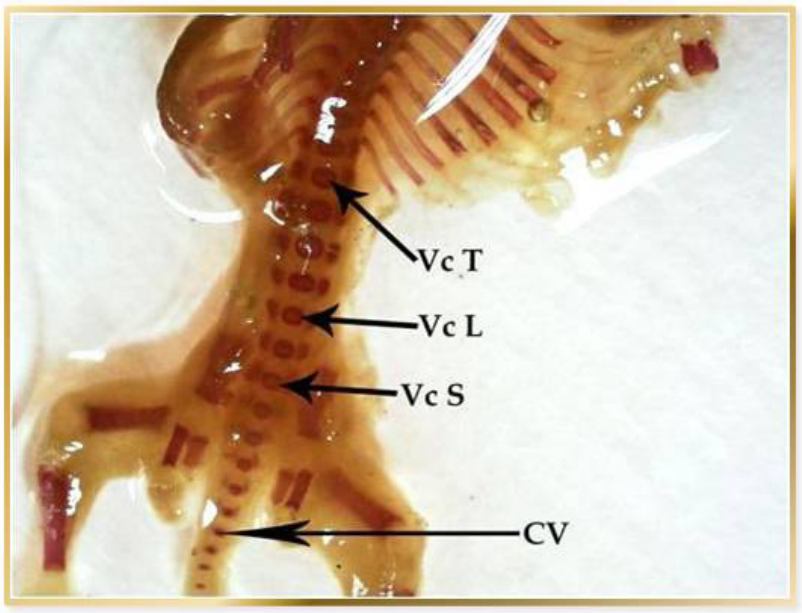

Fig. 8: Photomicrograph of fetal skeleton stained with alizarin red stain dorsal view in mouse fetus of negative control group taken by stereomicroscope (SZ 61)showing normal vertebral centra of thoracic $(\mathrm{Vc} T)$, lumbar( $\mathrm{Vc} L)$ and sacral vertebrae $(\mathrm{Vc}$ s). CV: caudal vertebrae. (X 10)

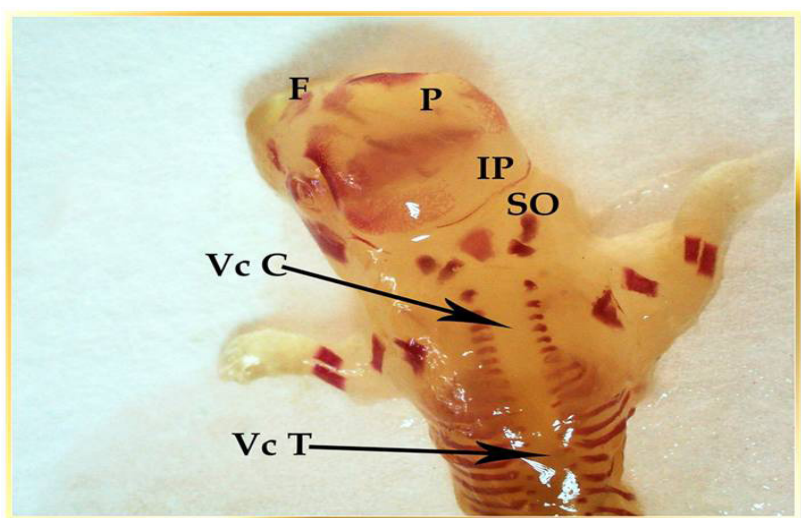

Fig. 9: Photomicrograph of fetal skeleton stained with alizarin red stain dorsal view in mouse fetus of group IV taken by stereomicroscope (SZ 61) showing poor ossification of skull bones and fore limb bones and absence of vertebral centra of cervical ( $\mathrm{VcC}$ ) and thoracic vertebrae ( $\mathrm{Vc} \mathrm{T})$ of group IV mouse fetus. F: frontal bone P: parietal bone. IP: tnterparietal bone. SO: suboccipital.(X 10)

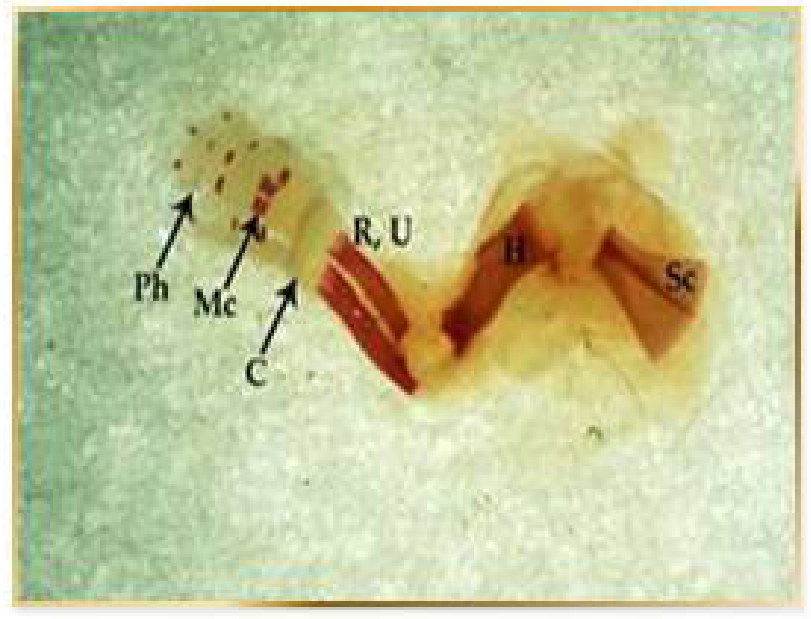

Fig. 10: Photomicrograph of fetal forelimb stained with alizarin red stain in mouse fetus of negative control group taken by stereomicroscope (SZ 61) showing normal ossification of fore limb bones. Sc: scapula. H: humerus. R: radius. U: ulna. C: carpals. Mc: metacarpals. Ph: phalanges. (X 12)

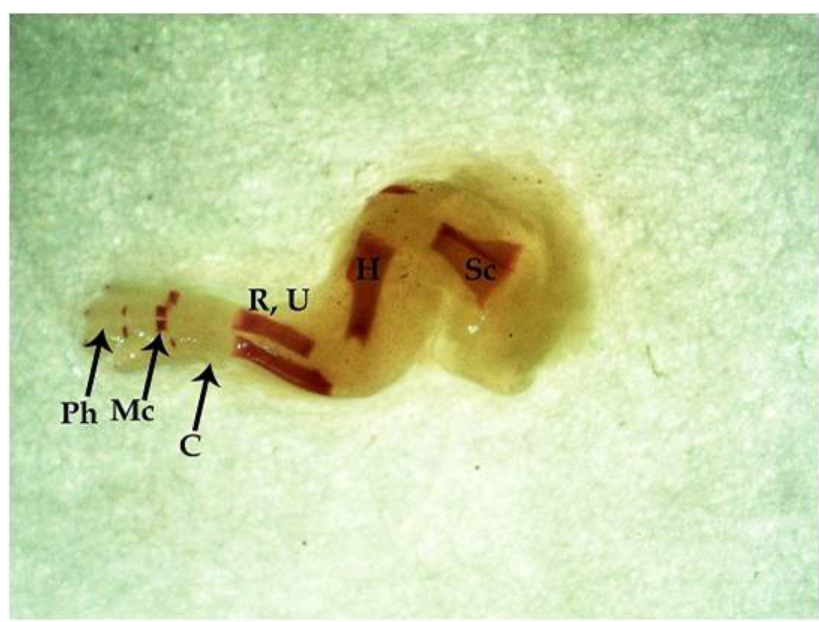

Fig. 11: Photomicrograph of fetal for limb stained with alizarin red stain in mouse fetus of group III taken by stereomicroscope (SZ 61) showing reduced ossification of fore limb bones. Sc: scapula $\mathrm{H}$ : humerus. R: radius. U: ulna. C: carpals. Mc: metacarpals. $\mathrm{Ph}$ : phalanges. (X 12)

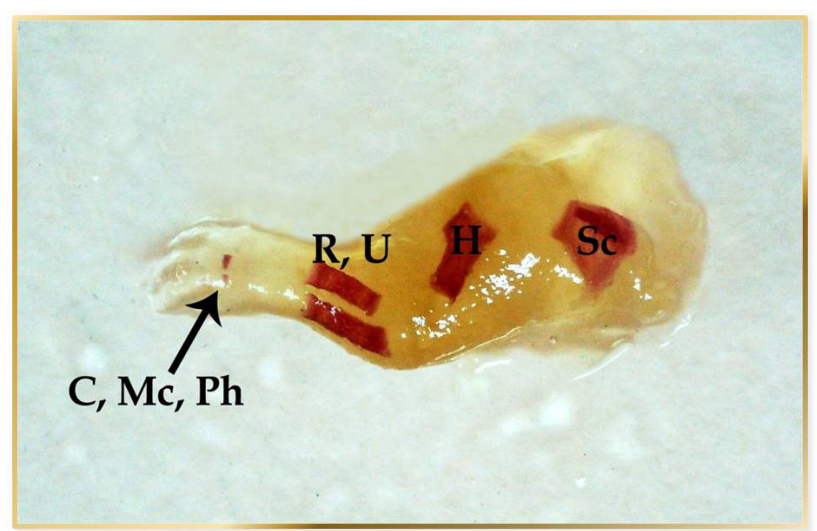

Fig. 12: Photomicrograph of fetal forelimb stained with alizarin red stain in mouse fetus of group IV taken by stereomicroscope (SZ 61) showing poor ossification of fore limb bones. Sc: scapula. $\mathrm{H}$ : humerus. R: radius. U: ulna. C: carpals. Mc: metacarpals. $\mathrm{Ph}$ : phalanges. (X 12) 


\section{DISCUSSION}

In the present study, administration of FA by the oral route during the period of organogenesis resulted in decrease in diet consumption, water intake, and weight of the pregnant mice. Intrauterine growth retardation and embryo feto-toxicity were observed as reduction in fetalweights, placental weights and number of live fetuses, and the higher incidences of resorption and post implantation death.

This can be due to formaldehyde's ability to rapidly cross placental barriers, so adversely affecting the mammalian fetal tissue ${ }^{[5]}$.

In the present study, there was a statistically significant reduction in the amount of food consumption in positive control group (group IB) and in the three FA treated groups (II, III and IV) compared to their corresponding negative control groups. This reduction appeared on day 9 of gestation and progressed through the gestational period.

Results of Saillenfait et $a l,{ }^{[17]}$ also support these results. As it reported reduction in food consumption of pregnant rats after inhalation of FA (10-40 ppm) .

On the other hand, Overman, ${ }^{[17]}$ noted that there was no significant reduction in maternal food and water consumption in pregnant hamsters exposed to percutaneous injection of $0.5 \mathrm{ml} \mathrm{FA} \mathrm{(37 \%} \mathrm{solution)} \mathrm{once}$ during gestational period.

There was a significant decrease in dams' weight gain between day 6 and day 18 in groups IB, III and IV, while the decrease in group II was insignificant compared to negative controls.

The decrease in maternal weight gain was due to reduction in diet consumption and water intake.

Saillenfait et al; ${ }^{[17]}$ reported that inhalation of FA (10-40 ppm) during the gestation period caused a significant decrease in body weight gain.

In contrast, Overman ${ }^{[18]}$ noted that there was no significant decrease in the weight of pregnant hamsters after injection of FA.

In the present study, there was no deaths associated with repeated FA exposure in group II and III, however $10 \%$ of group IV (FA $21 \mathrm{mg} / \mathrm{kg} / \mathrm{d}$ ) have died at GD16 which was insignificant. In contrast, significant elevation in the rate of death of pregnant mice was noted in the positive control group (group IB).

This is matching to Saillenfait et al, ${ }^{[17]}$ who reported that inhalation of FA in pregnant rats during the gestational period did not cause significant death of dams; In contrary to Marks et al ${ }^{[19]} 22 / 34$ pregnant mice died; on receiving oral FA at a dose of 74, 148, or $185 \mathrm{mg} / \mathrm{kg}$ /day on days 6 to 15 of gestation.

In the present study, the abortion rate increased insignificantly in groups II, III and IV, on comparison to negative controls. In contrast, there was significant increase in abortion rate in positive control group (group IB) by $60 \%$.

A retrospective cohort study of hospital staff in Finland found that formaldehyde exposure at sterilization units was not associated with an increase in $\mathrm{SAB}$, this results were depends on analysis of a small number of formaldehydeexposed women ( $\mathrm{n}=50$ exposed pregnancies) and 1100 unexposed pregnancies ${ }^{[20]}$.

These results does not match with the meta-analysis, by Collins et al, which included 8 epidemiology studies with data on occupational formaldehyde exposure and spontaneous abortion. Collins et al. reported there is a proof of increased risk of abortion with occupational formalin exposure ${ }^{[21]}$.

In the present study, the number of implants per litter was not altered significantly in groups II and III, but it was significantly decreased in group IV compared to negative control.

There was insignificant increase in the number of early resorptions in groups II, III and IV in comparison to negative control group. On the other hand, administration of FA at a dose of $21 \mathrm{mg} / \mathrm{kg} / \mathrm{d}$ on gestational days 6-13 in group IV resulted in significant increase in late resorption sites compared to the negative control, while the increase group II and III was insignificant.

The rate of live fetuses was significantly decreased in groups III and IV compared to the negative control. Also, the rate of dead fetuses was significantly increased in the same groups. However, administration of FA7/ kg /d at group II did not cause any significant change in the rate of live and dead fetuses.

These high incidences of post implantation deaths in the present study at a dose-dependent manner either as embryonic resorption or death can be correlated to the toxic effects of FA that occur in fetuses during the journey in the oviduct and during organogenesis leading to cytotoxic and teratogenic effects occurring after implantation.

These results agree with Fontignie et al who reported that embryo mortality, before and after implantation deaths increased significantly with paternal exposure by intraperitoneal injection of FA in male mice parents ${ }^{[22,23]}$.

Also, Thrasherand Kilburn ${ }^{[5]}$ in their 2001 study on pregnant rats have observed damage to blastomeres, increased rate of embryo degeneration; pre and postimplantation deaths increased by two fold; by inhalation of FA at 0.5 and $1.5 \mathrm{mg} / \mathrm{m} 34 \mathrm{~h} / \mathrm{d}$ for 4 months.

In the present study, a highly significant decline in fetal weight was observed in groups III and IV; however no significant change was noticed in group II.

Decrease in fetal body weight is a sensitive indicator for growth retardation. The possible cause may be due to maternal stress, which in turn affect the growing fetuses 
leading to reduced weight of the fetuses.

The reduced weight of the fetuses was accompanied by incomplete ossification of the fetal skeleton, which itself may be a factors that hinder growth leading to reduced weight of the fetuses.

However, Martin ${ }^{[24]}$ reported decrease in maternal food consumption, weight gain and decrease in bone ossification; but implantation sites, dead fetuses, fetal weights were all unaffected.

On the other hand, increased fetal body weight and increased overall dams' body weight was observed by Thrasher's 2001 study $^{[5]}$; in which, pregnant rats were exposed to inhalation of 0.5 and $1.5 \mathrm{mg} / \mathrm{m} 3 \mathrm{FA}, 4 \mathrm{~h} /$ day.

In the present study, reduction in placental weight took the same trend of fetal body weight. It was significantly decreased in groups III and IV, but group II showed no significant change compared to negative control.

A supporting study that reported decrease in placental weight and fetal body weight and live birth rate on exposure of wister rats to $0,5,25,120 \mathrm{mg} / \mathrm{m} 3 \mathrm{FA}$ for $1 \mathrm{~h} / \mathrm{d}$ for $7 \mathrm{wk}$ was done by Tang et al in $2006^{[25]}$.

In contrast, Thrasher ${ }^{[5]}$ in his above mentioned study reported no significant decrease in placental weight.

Congenital anomalies are characterized by physical, metabolic or anatomic deviations from the normal pattern of development that are apparent at birth and affect how a newborn will look and or function. They range from mild to fatal, and affect about $3 \%$ of all new borns in the US ${ }^{[26]}$.

Anomalies such as meningeocoele have been observed in the present study, this could be explained that FA can cause nervous system damage by its known ability to react with and form cross linking with proteins, DNA and unsaturated fatty acids ${ }^{[5]}$.

The occurrence of open eyelids in the present study were noted in some fetuses after FA administration, which may be due to incomplete ossification of skull bones that could affected the diameter of the orbit. This could result in changes in the attachment of eye muscles leading open eyelids as suggested by Saini et $a l^{[27]}$ who reported open eye lids after maternal exposure to nickel.

Findlater et $a l^{[28]}$, described eye lid development in the mouse. On GD 18, the eyes were completely covered by the fused eyelids. At birth; GD 22, the eyelids were tightly closed and remained so until $14 \mathrm{~d}$ after birth, when eyelid opening finally occurred.

Ophthalmic malformations was recorded after FA administration in fetuses of the present study. Anophthalmia $(1.31 \%, 3.07 \%$, and $13.15 \%$ in the three treated groups respectively) and microophthalmia $(2.28 \%, 1.95 \%$ and $15.78 \%$ in the three treatment groups respectively) were observed.

Ear abnormalities were observed such as absent ear (7.89\% in group IV), displaced ear (3.94, 3.07 and $18.42 \%$ in three treated groups) and deformed ear (1.53 and 10.53\% in groups III and IV).

To the best of our knowledge, these ophthalmic and ear abnormalities have not been mentioned in previous studies.

Abdominal wall defects such as omphalocele (3.07\% and $10.52 \%$ in group III and IV) and umbilical hernia $(3.95 \%, 10.77 \%$ and $18.42 \%$ in groups II, III and IV) were evident.

This agree with the results obtained by Al-Saraj ${ }^{[29]}$ who noticed umbilical hernia after administration of FA in pregnant female rabbits at concentration of 12 ppm FA by inhalation throughout the gestation period.

Another study done by Overman ${ }^{[30]}$ reported gastroschisis in the offspring of Golden Syrian hamsters $0.5 \mathrm{ml} \mathrm{FA}(37 \%)$ was applied to dorsal skin of pregnant hamsters for 2 h on GD 8, 9, 10, or 11 .

In the present study, significant increase in the rate of subcutaneous hemorrhage in cranial, abdominal, thoracic regions were observed in the fetuses of females treated with FA in group IV, compared to their corresponding negative control group.

These results correlates with Overman's study in which he reported increased incidence of subcutaneous hemorrhages after dams' topical exposure to $\mathrm{FA}^{[30]}$.

All the above mentioned congenital malformation can be explained that elimination of formaldehyde and its metabolites from fetal tissues is slower than maternal tissues. It has adverse effects on embryos which showed cytological injury and high rate of mortality, and its exposure throughout gestation caused a decreased DNA and RNA concentrations ${ }^{[5]}$.

FA is an alkylating agent so it has the ability to induce primordial germ cell mutations which resulted in fetal deaths and malformations; also it can induce addition and condensation reactions with proteins and amino acids as well as nucleic acids and nucleosides/tides, so it is regarded as a mutagen, cross linking agent and an immunogen ${ }^{[31]}$.

FA and alcohols probably affect embryos and the fetus via mitochondrial damage ${ }^{[31,32]}$. Oxygen stress, such as that caused by free radical generation, is associated with apoptotic cell death and fragmentation of mitochondrial genome ${ }^{[33]}$ Moreover, formaldehyde via FA generators, e.g. alkylating agents, initiates apoptosis ${ }^{[34]}$.

In the present study, Single stained skeletons of exposed fetuses revealed significant reduction in ossification of skull bones (nasal, frontal, parietal, interparietal, and supraoccipital), in groups II, III and IV.

In addition, reduced number of pelvic elements, reduced ossification/absence of carpals, metacarpals, tarsals, metatarsals, phalanges, and caudal vertebrae, were observed in groups II, III and IV.

However, significant displacement or absence of vertebral centra in thoracic, lumbar, and sacral regions was noted only in group IV. 
Exposure to various other environmental contaminants like chromium and cadmium was reported that it might produce similar effects ${ }^{[35]}$.

The decreased ossification of different bones could be due to change in calcium metabolism or decreased calcium and magnesium levels and /or altered calcitonin level in the growing fetus, causing abnormalities in bone development ${ }^{[23]}$.

\section{CONCLUSIONS}

Formaldehyde showed maternal and fetotoxicity at a maternal dose of $14 \& 21 \mathrm{mg} / \mathrm{kg}$ per day.

FA caused signs of CNS toxicity including lethargy, decrease in motor activity, and loss of appetite in dams throughout the period of treatment.

Maternal weight gain was also significantly reduced among the entire period of gestation 6- 18 gestational days occurring simultaneously with reduction in food consumption indicating maternal toxicity or embryo lethality.

Increased number of late resorptions, dead fetuses and fetal weight reduction indicates its embryotoxicity and fetotoxicity.

FA caused external and skeletal abnormalities indicating that FA is a teratogenic agent during the period of organogenesis.

\section{CONFLICTS OF INTEREST}

There are no conflicts of interest

\section{REFERENCES}

1. World Health Organization international agency for research on cancer. IARC Monographs on the Evaluation of Carcinogenic Risks to Humans. Volume 87. Lyon, France: IARC Library Cataloguing in Publication Data; 2006.

2. Tokars JI, Miller ER, Alter MJ, Arduino MJ. National Surveillance of Dialysis-associated Diseases in the United States, 1997. Atlanta, GA: National Center for Infectious Diseases (NIC), Centers for Disease Control and Prevention (CDC); 2000.

3. Rademaker M. Contact dermatitis to phenolformaldehyde resin in two plywood factory workers. Australas J Dermatol 2002; 43(3):224-5.

4. Nakao R. Formaldehyde-induced shrinkage of rat thymocytes. J Pharmacol Sci 2003; 91(1): 83-6.

5. Thrasher JD, Kilburn KH. Embryo toxicity and teratogenicity of formaldehyde. Arch Environ Health 2001; 56(4): 300-11.

6. Rumchev KB. Domestic exposure to formaldehyde significantly increases the risk of asthma in young children. Eur Respir J 2002; 20(2):403-8.
7. Yang X. Eye irritation caused by formaldehyde as an indoor air pollution - a controlled human experiment. Biomed Environ Sci 2001; 14(3): 229-36.

8. Nielsen GD, Wolkoff P. Cancer effects of formaldehyde: a proposal for an indoor air guideline value. Arch Toxicol 2010; 84(6):423-46.

9. Cheng G, Shi Y, Sturla SJ, Jalas JR, Mclntee EJ, Villalta PW. Reactions of formaldehyde plus acetaldehyde with deoxyguanosine and DNA: Formation of cyclic deoxyguanosine adducts and formaldehyde cross- links. Chem Res Toxicol 2003; 16:145-52.

10. Metz B, Kersten GF, Hoogerhout P, Brugghe HF, Timmermans HA, de Jong A. Identification of formaldehyde - induced modifications of proteins: reactions with model peptides. J Biol Chem 2004; 279: 6235-43.

11. Ye X, Yan W, Xie H, Zhao M, Ying C. Cytogenetic analysis of nasal mucosa cells and lymphocytes from high - level long- term formaldehyde exposure and low - level short -term exposed waiters. Mutat Res 2005; 588:22-7.

12. Tyrkiel E, Wiadrowska B, Ludwicki JK. Induction of micronuclei in erythrocytes of bone marrow and peripheral blood in laboratory mice following acute and subchronic exposure to DDT (fenarimol and nuarimol). Rocz Panstw Zakl Hig 1996; 47(2):151-8.

13. Wilson JG, Warkany J. Evalution of skeleton In: Wilson JG, Warkany J. (eds). Teratology. Principles and techniques. In: A compendium of lectures and demonstrations from the first workshop in teratology, held February 2-8, 1964, at the College of Medicine of the University of Florida. Sponsored by the Commission on Drug Safety. Chicago \& London: University of Chicago Press; 1965. 145-8.

14. Reynaud L, Jocteur-Monrozier A. Skeletal examination by alizarin staining. Methods Mol Biol 2013; 947:201-13.

15. Altman GA. Practical Statisyics for Medical Research, 1st ed. London: Chapman \& Hall; 1991

16. Ramos-Remus C, Dorazco-Barragan G, Aceves-Avila FJ, Alcaraz-Lopez F, Fuentes-Ramirez F, Michel-Diaz $\mathrm{J}$, et al. Genotoxicity assessment using micronuclei assay in rheumatoid arthritis patients. Clin Exp Rheumatol 2002; 20(2): 208-12.

17. Duong A, Steinmaus C, McHale CM, Vaughan CP, Zhang L. Reproductive and developmental toxicity of formaldehyde: A systematic review. Mutat Res 2011; 728(3):118-38

18. Overman DO. Absence of embryotoxic effects of formaldehyde after percutaneous exposure in hamsters. Toxicol Lett 1985; 24: 107-10. 
19. Marks TA, Worthy WC, Staples RE. Influence of formaldehyde and Sonacide (potentiated acid glutaraldehyde) on embryo and fetal development in mice. Teratology 1980; 22(1):51-8.

20. Hemminki K, Mutanen P, Saloniemi I. Spontaneous abortions in hospital staff engaged in sterilising instruments with chemical agents. Br Med J 1982; 285:1461-3.

21. Collins JJ, Ness R, Tyl RW, Krivanek N, Esmen NA, Hall TA. A Review of Adverse Pregnancy Outcomes and Formaldehyde Exposure in Human and Animal Studies.Regul Toxicol Pharmacol 2001; 34:17-34.

22. Fontignie-Houbrechts N. Genetic effects of formaldehyde in the mouse. Mutat Res 1981; 88(1):109-14.

23. Fontignie-Houbrechts N, Moutschen-Dahmen $M$, Degraeve N, Gloor H. Genetic effects in the mouse of formaldehyde in combination with adenosine and hydrogen peroxide. Mutat Res 1982; 104(6):371-6.

24. Martin WJ. A teratology study of inhaled formaldehyde in the rat. Reprod Toxicol 1990; 4:237-9.

25. Tang QN, Hao JG, Xia Q, Ru JG, Zhang C, Huang YF. Toxic effect of formaldehyde on reproduction and development of rats. Endemic Dis Bull 2006; 21: $15-8$.

26. Dzierzawski A.Embryotoxic and teratogenic effects of phenylmercuric acetate and methylmercuric chloride in hamsters, rats and rabbits. Pol Arch Weter 1979; 22(2):263-87.

27. Saini S, Nair N, Saini MR. Embryotoxic and teratogenic effects of nickel in Swiss albino mice during organogenetic period. Biomed Res. Int 2013;2013:701439.

28. Findlater GS, McDougall RD, Kaufman MH. Eyelid development, fusion and subsequent reopening in the mouse. J Anat 1993; 183 (Pt 1):121-9.

29. Al-Saraj AA. Teratogenic effect of formaldehyde in rabbits. Iraqi J Vet Sci 2009; 23: 1-4.

30. Overman DO. Testing for percutaneous embryotoxicity of laboratory reagents in the hamster. Teratology 1981; 23(2): 56A.

31. Speit G, Schutz P, Merk O. Induction of formaldehydeinduced DNA protein crosslinks in repair-deficient human cell lines. Mutagenesis 2000; 15:85-90.

32. Ikonomidou C, Bittigau P, Ishimuaru, MJ, Wozniak DF. Ethanol induced apoptotic neurodegeneration and fetal alcohol syndrome. Science2000; 287: 1056-60.

33. Yoneda M, Katsumata K, Hayakawa M. Oxygen stress induces apoptotic cell death associated with fragmentation of mitochondrial genome. Biochem Biophys Res Comm1995; 209: 723-9.

34. Szende B, Tyihak E, Trezly L. Formaldehyde generators and capturers as influencing factors of mitotic and apoptotic processes. Acta Biol Hung1998; 49: 323-9.

35. Marouani N, Tebourbi O, Mokni M, Yacoubi MT, Sakly M, Benkhalifa M, et al. Embryotoxicity and fetotoxicity following intraperitoneal administrations of hexavalent chromium to pregnant rats. Zygote 2011; 19(3):229-35. 


\section{الملخص العربى}

\section{تقييم التشوه الجنينى للقورمالين على اجنة الفئران البيضاء الحوامل ميلاد نعيم بشرى قلادة'، فردوس أحمد القلعة'، علياء محمد توفيق عبد المطلب العلقامى' مجدى ممدوح حسن ألبردينى"}

'ققم التشريح، ‘قتم الباثولوجيا الاكلينيكية ـ كلية الطب - جامعة الإسكندرية

المقدمه: الفور مالد هيد مادة كيميائية مهمة اقتصاديا يستخدم فى صناعة الادوات المنزلية ومستحضر ات التجميل وبعض المستحضر ات الطبية ولكنه مادة مسرطنة ومحفزة لحدوث الطفر ات هيداته

التعرض لمادة الفورمالدهيد شائع بين المشتخلين بالمهن الطبية حيث انه يستخدم على نطاق و اسع كمطهر. التعرض للفورمالين يبدو جليا واجبارى بين العمال و العاملين بأقسام التشريح بكليات الطب على مستوى الجمهورية؛ حيث يستخدم كمادة اساسية لحفظ الجثث و العينات التشريحية. العاملين بأقسام التشريح هم بين اكثر الاشخاص تعرضا لمادة الفورمالين ولذلك هم الاكثر عرضة للمخاطر الصحية لتلك المادة، مما يستلزم عمل در اسات بحثية متكاملة للمخاطر الصحية لتلك المادة وخاصة تأثثر ها المحتمل على أجنة

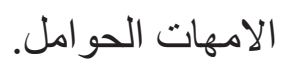

الهدف من البحث: لقد صممت الدر اسية الحلية لتقييم التأثير التشويهى للفورمالين على حيوانات التجارب. مواد وطرق البحث: تم الحصول على • • إناث الفئر ان الحوامل. تم تقسيمهم إلى خمس مجموعات: 1. مجمو عة ضابطة سلبية: شملت · ( من الإناث الحوامل وقد تلقت الماء المقطر من اليوم السادس الي اليوم لثالث

$$
\text { عشر من الحمل. }
$$

2. مجمو عة ضابطة إيجابية: شملت · / من الإناث الحو امل وقد تلقت الكولثيسين عند مستوى جر عة ب مجم / كجم /

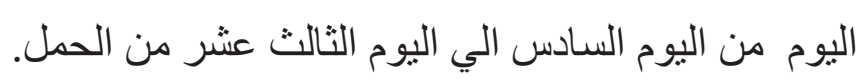

3. مجموعة شملت • 1 من الإناث الحو امل وقد تلقت الفورمالدهيد عند مستوى جرعة V مجم / كجم /اليوم من اليوم

$$
\text { السادس الي اليوم الثالث عثر من الحمل. }
$$

4. مجموعة شملت ـ من الإناث الحوامل وقد تلقت الفورمالدهيد عند مستوى جرعة ؟ ا مجم / كجم /اليوم من اليوم

$$
\text { السادس الي اليوم الثالث عشر من الحمل. }
$$

5. المجموعة شملت · ( من الإناث الحوامل وقد تلقت الفورمالدهيد عند مستوى جرعة اب مجم / كجم /اليوم من

$$
\text { اليوم السادس الي اليوم الثالث عشر من الحمل. }
$$

تم التضحية بالإناث الحو امل فى اليوم الثامن عشر من الحمل وتم فحص الرحم واستخر اج الأجنة و فحصها مجهريا. 
النتائج: اظهرت النتائج الاتى: نقص فى وزن الاجنة، زيادة معدلات الاجهاض ومعدلات وفيات المو اليد، وظهرت

بعض العيوب الخلقية مثل: الفتاء السرى، نزيف تحت الجلد، تشوهات بالاذن، تأخر تعظم عظام الجمجمة والفقرات الظهرية.

الاستتتاج: النتائج السابقة ترجح كون الفورمالين مادة مشوهة. 